\title{
Branched-chain Amino Acid Supplement
}

National Cancer Institute

\section{Source}

National Cancer Institute. Branched-chain Amino Acid Supplement. NCI Thesaurus. Code C120121.

A nutritional supplement containing essential branched-chain amino acids (BCAAs), including leucine, isoleucine and valine, with potential anti-cachectic, antiangiogenic, hepatocellular carcinoma (HCC) inhibiting and hepatoprotective activities. Upon oral administration, BCAAs inhibit the expression of both hypoxia-inducible factor 1-alpha subunit (HIF-1a) and vascular endothelial growth factor (VEGF), which prevents VEGFmediated ang iogenesis in HCC cells. In addition, BCAAs inhibit proliferation and induce apoptosis of HCC cells by both suppressing the expression of insulin-like growth factor (IGF), and inhibiting the phosphatidylinositol 3-kinase (PI3K)/Akt/mammalian target of rapamycin (mTOR) pathway. BCAAs also correct the plasma amino acid imbalance and promote protein metabolism, including the synthesis of albumin and glycogen. They reduce oxidative stress by inducing the activation of genes involved in antioxidant defenses, which prevent the production of reactive oxygen species (ROS). BCAAs also strengthen the immune system by increasing hepatic lymphocytes and stimulating natural killer (NK) cell activity. This supplement is able to improve insulin resistance and promote ammonia detoxification through increased glutamine (Gln) production. 